\title{
Regulation of IL-6 signaling by miR-125a and let-7e in endothelial cells controls vasculogenic mimicry formation of breast cancer cells
}

\author{
Youngsook Park ${ }^{1} \mathcal{E}$ Jongmin Kim ${ }^{1,2, *}$ \\ ${ }^{1}$ Division of Biological Sciences, Sookmyung Women's University, Seoul 04310, ${ }^{2}$ Research Institute for Women's Health, Sookmyung \\ Women's University, Seoul 04310, Korea
}

\begin{abstract}
The role of tumor-proximal factors in tumor plasticity during chemoresistance and metastasis following chemotherapy is well studied. However, the role of endothelial cell (EC) derived paracrine factors in tumor plasticity, their effect on chemotherapeutic outcome, and the mechanism by which these paracrine factors modulate the tumor microenvironment are not well understood. In this study, we report a novel mechanism by which endothelial miR-125a and let-7e-mediated regulation of interleukin-6 (IL-6) signaling can manipulate vasculogenic mimicry (VM) formation of MDA-MB-231 breast cancer cells. We found that endothelial IL-6 levels were significantly higher in response to cisplatin treatment, whereas levels of IL-6 upon cisplatin exposure remained unchanged in MDA-MB-231 breast cancer cells. We additionally found an inverse correlation between IL-6 and miR-125a/let-7e expression levels in cisplatin treated ECs. Interestingly, IL-6, IL-6 receptor (IL-6R), and signal transducer and activator of transcription 3 (STAT3) genes in the IL-6 pathway are closely regulated by miR-125a and let-7e, which directly target its $3^{\prime}$ untranslated region. Functional analyses revealed that endothelial miR-125a and let-7e inhibit IL-6-induced adhesion of monocytes to ECs. Furthermore, conditioned medium from cisplatin treated ECs induced a significantly higher formation of VM in MDA-MB-231 breast cancer cells as compared to that from intact ECs; this effect of cisplatin treatment was abrogated by concurrent overexpression of miR-125a and let-7e. Overall, this study reveals a novel EC-tumor cell crosstalk mediated by the endothelial miR-125a/let-7e-ll-6 signaling axis, which might improve chemosensitivity and provide potential therapeutic targets for the treatment of cancer. [BMB Reports 2019; 52(3): 214-219]
\end{abstract}

*Corresponding author. Tel: +82-2-710-9553; Fax: +82-2-20777322; E-mail: jkim@sookmyung.ac.kr

https://doi.org/10.5483/BMBRep.2019.52.3.308

Received 19 December 2018, Revised 4 January 2019, Accepted 8 January 2019

Keywords: Chemoresistance, Endothelial cell, IL-6 signaling, microRNA, Vasculogenic mimicry

\section{INTRODUCTION}

Although many chemotherapeutic drugs have received remarkable initial responses, tumor recurrence is common. Chemoresistance and metastasis are the most common causes of cancer related death (1-3). In light of the limitations of current chemotherapeutic drugs, identification of novel mechanisms to overcome tumor recurrence, chemoresistance, and metastasis is critical.

To date, most studies have focused on the role of tumorproximal factors in tumor recurrence and chemoresistance following chemotherapy (1, 4-6). Despite the support provided by the surrounding tumor microenvironment to cancer cells, the role of tumor stromal cells such as endothelial cells (ECs) in chemotherapeutic outcomes is still poorly understood. Healthy vascular ECs play an essential role in the maintenance of vascular homeostasis in response to various stimuli (7-9). In contrast, endothelial dysfunction caused by chemotherapeutic drugs contributes to tumor growth and survival $(10,11)$. However, it is not well understood how EC-derived paracrine factors affect endothelial dysfunction following chemotherapy, and how they influence tumor plasticity which eventually causes chemoresistance. Vasculogenic mimicry (VM) refers to the formation of tumor cell-lined endothelium-like vessels, which is known to provide blood supply for tumor growth and represents a key link connecting the aggressiveness of cancer and metastasis with short survival of patients (12-15). In addition, emerging evidence shows that VM plays key roles in tumor plasticity during drug resistance following chemotherapy $(16,17)$.

Interleukin-6 (IL-6) signaling plays key roles in the development of chemoresistance and in recurrence of various cancers. (18-20). Thus, IL-6, IL-6 receptor (IL-6R), signal transducer and activator of transcription 3 (STAT3), and gp130 genes in the IL-6 signaling pathway have been considered as key therapeutic targets for the treatment of many cancers (18-21). IL-6 is secreted by tumor cells or surrounding tumor microenvironments, such as ECs, macrophages and fibroblasts, and, orchestrates tumor progression in an autocrine or paracrine manner $(11,21)$. While many studies have demonstrated that the role 
of tumor cell-derived IL-6 is associated with chemoresistance in various tumor types, relatively few studies have investigated the role of EC-derived IL-6 in tumor plasticity and its effect on chemotherapeutic outcome. In addition, it is not clear whether any microRNA (miRNA) mediated regulatory mechanisms are involved in IL-6 production and whether they have an effect on chemotherapeutic outcome in ECs.

In this study, we sought to define the role of the EC-derived paracrine factor, IL-6 in tumor plasticity in a chemotherapeutic therapy, and to understand the role of miRNAs in IL-6 signaling mediated paracrine effects on tumor cells. We demonstrated that IL-6 levels are upregulated as a consequence of decrease in miR-125a and let-7e expression in cisplatin treated ECs, although IL-6 levels were not affected in cisplatin treated MDA-MB-231 breast cancer cells, which suggests the importance of endothelium-derived IL-6. We found that conditioned medium (CM) from cisplatin treated ECs induces significantly greater formation of VM in MDAMB-231 breast cancer cells, and might eventually cause drug resistance and extensive metastasis. These findings reveal a novel mechanism by which miRNA-mediated regulation of endothelial IL-6 signaling can manipulate VM formation through the platinum-containing chemotherapeutic agent, cisplatin in breast cancer cells.

\section{RESULTS}

\section{Cisplatin treatment induces IL-6 expression and inhibits miR-125a/let-7e expression in ECs}

To analyze the effect of a chemotherapeutic drug on IL-6 expression in the tumor microenvironment, we treated tumor cells and ECs with cisplatin and assessed expression levels of IL-6. Treatment of human umbilical vein ECs (HUVECs) with cisplatin led to significantly increased IL-6 expression levels (Fig. 1A), whereas IL-6 expression was not affected in cisplatin treated MDA-MB-231 breast cancer cells (Data not shown), suggesting a potential role for endothelium-derived paracrine IL-6 secretion in the microenvironment of breast cancer. Several molecular mechanisms have been shown to affect IL-6 expression, including a critical role for miRNA-mediated regulation in different cellular contexts, such as in macrophages (22) and preosteoblasts (23). The potential role of miRNAs as regulators of IL-6 expression in ECs is still unknown. Thus, to identify miRNAs that might regulate the expression of IL-6 in response to cisplatin in ECs, we first analyzed the expression of miRNAs in response to cisplatin in HUVEC and EC-enriched miRNAs, using publicly available miRNA expression profiling data $(22,23)$. The target prediction algorithm (TargetScan) was used to identify potential miRNAs targeting IL-6 and related genes (Fig. 1B). From these results, we found two miRNAs (miR-125a and let-7e) which are highly expressed in ECs and are expressed as a cluster. We next examined miR-125a and let-7e expression in cisplatin treated ECs. As shown in Fig. 1C, miR-125a and let-7e levels decreased in response to cisplatin treatment in ECs. These findings suggest that miR-125a and let-7e may be involved in the mechanism underlying the induction of IL-6 expression in response to cisplatin.

\section{IL-6, IL-6R and STAT3 genes from the IL-6 pathway are directly targeted by miR-125a/let-7e, and these miRNAs affect the phosphorylation of STAT3}

To further investigate the relationship between miR-125a/ let-7e and IL-6, we determined the effects of miR-125a and let-7e overexpression on IL-6 expression in HUVECs. Overexpression of miR-125a or let-7e led to significant downregulation of IL-6 expression, whereas inhibition of endogenous miR-125a or let-7e by anti-miRs in HUVECs led to upregulation of IL-6 expression (Fig. 2A). Next, we determined whether miR-125a and let-7e regulate IL- 6 expression by binding directly to its $3^{\prime} U T R$. Let-7e was predicted to target the IL-6 3'UTR, while miR-125a was not (Fig. 1B). We generated luciferase constructs containing the $3^{\prime} \cup T R$ of $I L-6$ and found that let-7e overexpression led to a marked decrease in reporter activity. This response was abrogated with mutagenized IL-6 3'UTR (Fig. 2B). Overall, these results show that let-7e can directly target $I L-6$. However, further investigation is required to elucidate the molecular mechanism by which miR-125a inhibits IL-6 expression.

We further examined additional predicted targets of let-7e and miR-125a which might play an essential role in the IL-6 signaling pathway in ECs. As shown in Fig. $2 \mathrm{C}$ and 2D,

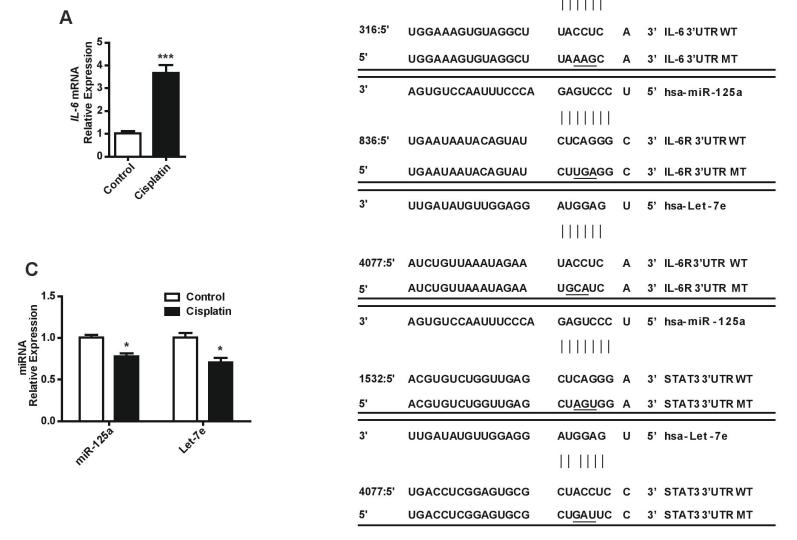

Fig. 1. Cisplatin treatment upregulates IL-6 and reduces miR-125a/ let-7e expression in HUVECs. (A) IL-6 mRNA expression in response to cisplatin treatment $(10 \mu \mathrm{M})$ in HUVECs. (B) Predicted target sequences of IL-6, IL-6R, and STAT3 3'UTRs targeted by miR-125a and let-7e, and mutated sequences for disrupting miR$125 \mathrm{a}$ and let-7e recognition sequences. (C) Quantitative PCR showing expression of miR-125a and let-7e in response to cisplatin treatment $(10 \mu \mathrm{M})$ in HUVECs. $* P<0.05$, ***P $<0.001 \mathrm{com}-$ pared to controls. Error bars, s.e.m. 
overexpression or knockdown of miR-125a or let-7e had effects on IL-6R and STAT3 expression similar to those on IL-6 expression, although let-7e had no effect on STAT3 mRNA levels, and we demonstrated that miR-125a and let-7e significantly reduced reporter activity of the $3^{\prime} U T R$ of IL-6R and STAT3, though not that of the mutant forms (Fig. 2E). Finally, we speculated whether perturbation of miR-125a and let-7e expression affects the phosphorylation of STAT3, a key downstream mediator of IL-6 signaling (24). miR-125a and let-7e overexpression led to a robust decrease in STAT3
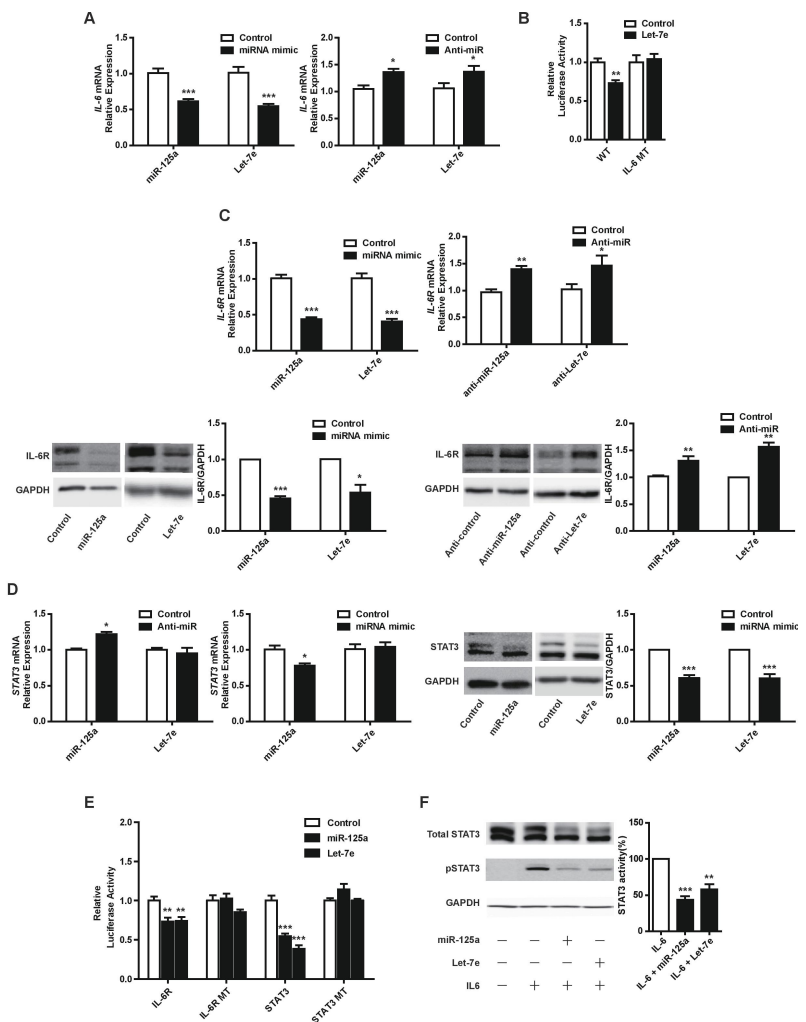

Fig. 2. miR-125a and let-7e inhibit IL-6 signaling by directly targeting the $I L-6, I L-6 R$ and STAT3 genes in HUVECs. (A) IL-6 mRNA expression in response to overexpression of miR-125a or let-7e mimics, or inhibition of miR-125a or let-7e with anti-miRs in HUVECs. (B) Effect of let-7e overexpression on the luciferase reporter containing either the wild-type or mutagenized 3'UTR of the human IL-6 gene in HEK-293 cells. Luciferase activity for constructs with the wild-type (WT) and mutant (MT) 3'UTR sequences. (C, D) IL-6R and STAT3 expression in response to overexpression of miR-125a or let-7e mimics, or inhibition of miR-125a or let-7e with anti-miRs in HUVECs. (E) Effect of miR-125a or let-7e overexpression on the luciferase reporter containing either the wild-type or mutagenized 3'UTR of the human IL-6R or STAT3 gene in HEK-293 cells. Luciferase activity for constructs with the WT and MT 3'UTR constructs. (F) STAT3 phosphorylation in response to IL-6 $(100 \mathrm{ng} / \mathrm{ml})$ in HUVECs with concurrent overexpression of miR-125a or let-7e. $* \mathrm{P}<0.05$, $* * \mathrm{P}$ $<0.01, * * * \mathrm{P}<0.001$ compared to controls. Error bars, s.e.m. phosphorylation with exogenous IL-6 stimulation (Fig. 2F).

\section{Endothelial miR-125a/let-7e suppresses IL-6-induced adhesion of monocytes to ECs and induces paracrine inhibition of VM formation by cisplatin-treated EC} conditioned media

Because the IL-6 signaling pathway plays a critical role in vascular functions such as inflammation and monocyte adhesion $(25,26)$, we examined the effect of miR-125a/let-7e on IL-6 induced adhesion of monocytes to ECs. miR-125 and let-7e overexpression significantly inhibited the IL-6 induced THP-1 cell adhesion to HUVECs (Fig. 3A), suggesting that this effect may be secondary to the targeting of IL-6R and STAT3. However, miR-125 and let-7e overexpression had no effect on

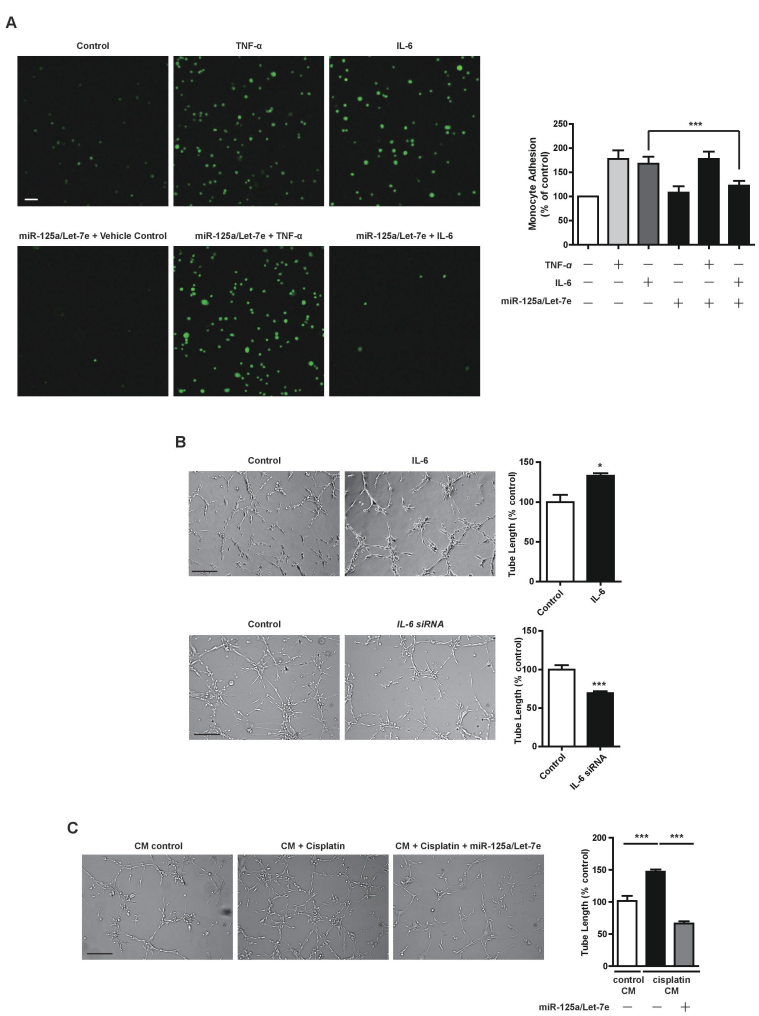

Fig. 3. Endothelial miR-125a/let-7e axis suppresses IL-6-induced adhesion of monocytes to ECs and regulates the development of vasculogenic mimicry in MDA-MB-231 breast cancer cells in a paracrine manner. (A) IL-6 recombinant protein $(100 \mathrm{ng} / \mathrm{ml})$ increases adhesion of monocytes to HUVECs and miR-125a/let-7e overexpression suppresses IL-6-induced adhesion of monocytes to HUVECs. (B) Development of vasculogenic mimicry in response to $\mathrm{IL}-6$ recombinant protein $(100 \mathrm{ng} / \mathrm{ml})$ or $/ L-6$ knockdown in MDA-MB-231 breast cancer cells. (C) Development of vascular mimicry of MDA-MB-231 breast cancer cells induced by cisplatin treated EC conditioned medium (CM) with concurrent overexpression of miR-125a and let-7e. $* P<0.05, * * * P<0.001$ compared to controls. Scale bar, $200 \mu \mathrm{m}$. Error bars, s.e.m. 
the TNF- $\alpha$ induced THP-1 cell adhesion to HUVECs. These results indicate that miR-125 and let-7e might act specifically on the adhesion of monocytes to ECs in response to IL-6.

Next, to understand the role of EC derived paracrine IL- 6 on tumor plasticity, we treated MDA-MB-231 breast cancer cells with IL-6, and examined the formation of VM using the well established in vitro tube formation assay. As shown in Fig. 3B, IL-6 significantly induced the formation of VM in MDA-MB231 breast cancer cells, whereas IL-6 knockdown led to a significant decrease in VM formation. Given that cisplatin treatment induces IL-6 expression and inhibits miR-125a/let-7e expression in ECs, we evaluated the role of IL- 6 regulation by miR-125a and let-7e on tumor plasticity in response to cisplatin, and we subjected MDA-MB-231 breast cancer cells to control EC conditioned medium (CM) or cisplatin containing EC CM. We found that treatment with cisplatin containing EC $\mathrm{CM}$ resulted in a robust increase in $\mathrm{VM}$ formation compared to treatment with $\mathrm{CM}$ from control cells. This effect of cisplatin treatment was abrogated by concurrent overexpression of miR125a and let-7e (Fig. 3C). Altogether, IL-6 signaling regulation by miR-125a and let-7e in ECs might modulate endothelial dysfunction and VM formation upon cisplatin treatment.

\section{DISCUSSION}

Previous studies have associated elevated IL-6 levels with poor overall survival and tumor progression in various cancers (27-29). In addition, IL-6 signaling plays a key role in the development of chemoresistance in various cancers (18-20). Vasculature is essential for cancer progression and reprograms the tumor microenvironment. In particular, ECs play a critical role in the maintenance of vascular homeostasis, whereas dysfunctional ECs contribute to the pathogenesis of various diseases and to tumor progression by secreting cytokines. (10, 11). Here, we describe miRNA-driven regulation of IL-6 signaling in ECs and show its involvement in the regulation of cell adhesion and in the formation of VM in an autocrine and paracrine manner in tumor microenvironments in response to cisplatin treatment.

Many studies have shown that miRNAs play key roles in the context of cancer and vascular biology (9, 10, 30-33). However, endothelial miRNA-mediated autocrine and paracrine regulation of chemotherapeutic outcome in tumor microenvironments is poorly understood. Previous reports show that miR-125a and let-7e are significantly downregulated in many cancers, and downregulation of these miRNAs leads to enhanced tumor progression, chemoresistance, and metastasis despite strong context dependence of the role of miR-125a and let-7e in cancer (34-40). Although miR-125a and let-7e are highly expressed in ECs $(8,41)$, and have been studied in different cellular contexts such as in immune cells, preosteoblasts, and in a variety of cancers $(23,24,34,37,42,43)$, their role in EC biology remains unclear. Therefore, it is important to understand the role or regulation of miR-125a and let-7e in tumors as well as in tumor microenvironments such as ECs. Our current findings provide three key aspects of highly enriched endothelial miRNAs, miR-125a and let-7e, which might act as important mediators for modulating tumor plasticity and thus affect the chemotherapeutic outcome. First, we found that IL-6 and miR-125a/let-7e are inversely regulated by the platinum-containing chemotherapeutic agent, cisplatin in HUVECs. Second, we showed that miR-125a and let-7e directly target IL-6, IL-6R and STAT3 genes from the IL-6 pathway and inhibit IL-6-induced adhesion of monocytes to ECs. Lastly, we found that miR-125a and let-7e suppress VM formation in MDA-MB-231 breast cancer cells using CM from cisplatin treated EC cultures by targeting IL- 6 signaling. Together, our findings support the hypothesis that endothelium mediated paracrine cancer regulation affects chemotherapeutic outcome, and provide a novel molecular mechanism by which miRNA mediated EC paracrine factors modulate VM formation in breast cancer cells.

VM reflects tumor plasticity of aggressive tumor cells acquiring tumor cell-lined endothelium-like vessels, and is an independent blood supply system from endothelial vessels. Emerging evidence shows that the roles of VM in several cancers, are associated with tumor aggressiveness and poor patient survival, as well as tumor plasticity during chemoresistance and metastasis (12-17). In light of the association of VM and tumor progression, coupled with ineffective antiangiogenic drugs associated with the inhibition of VM (44, 45), identifying novel therapeutic targets that inhibit VM, might be effective to overcome chemoresistance and to eliminate tumor progression. However, the molecular mechanism underlying the formation of VM in tumor microenvironments remains unclear. Our current data provide a novel mechanism by which restoration of miR-125a and let-7e expression in cisplatin treated ECs can inhibit VM formation in MDA-MB-231 breast cancer cells, in a paracrine manner via targeting IL-6 signaling in ECs. These findings support the emerging role of dysfunctional EC-tumor cell crosstalk which likely plays a critical role in tumor progression and chemoresistance. Given these findings, future studies will be necessary to identify other paracrine factors in ECs affecting chemotherapeutic outcome. Furthermore, our ongoing studies to elucidate the precise role of miR-125/let-7e-IL-6 signaling axis in ECs, both in chemotherapeutic drug induced endothelial dysfunction, and tumor progression in a paracrine manner, will provide greater insights into tumor-EC interaction.

In conclusion, we show that downregulation of miR-125a and let-7e and the subsequent activation of IL-6 signaling in ECs on chemotherapeutic drugs is closely associated with cisplatin-induced VM formation and monocyte adhesion in tumor microenvironments. These findings might promote the development of novel therapeutic candidate drugs, such that chemotherapy combined with IL- 6 inhibition might help to overcome chemoresistance and, thus, help in treatment of cancer. 


\section{MATERIALS AND METHODS}

An extended Materials and Methods section is available in the Supplementary Material.

\section{Cell culture and transfection}

HUVECs (Human umbilical vein ECs; Lonza and Yale VBT Core) were maintained in EBM-2 basal medium supplemented with EGM-2 (Lonza) with 1\% penicillin/streptomycin (Welgene). HUVECs were grown to $70 \%$ to $90 \%$ confluency and used between passages 4 to 7 for all experiments. MDA-MB-231 (human breast adenocarcinoma cells) were maintained in DMEM (Dulbecco's modified Eagles medium; Welgene) supplemented with $10 \%$ FBS (fetal bovine serum, Hyclone) and $1 \%$ penicillin-streptomycin (Welgene). HEK-293 cells (human embryo kidney cells) were maintained in DMEM supplemented with $10 \% \mathrm{FBS}$ and $1 \%$ penicillin/streptomycin. Cells were maintained in a $5 \% \mathrm{CO}_{2}$ incubator at $37^{\circ} \mathrm{C}$. siRNA (Bioneer), miRNA mimics and anti-miRs (miRVana; Ambion) were transfected into cells using Lipofectamine RNAiMAX (Invitrogen) according to the manufacturer's instructions.

\section{ACKNOWLEDGEMENTS}

This work was supported by the Medical Research Center Program (No. 2011-0030074) through National Research Foundation of Korea (NRF) grant funded by the Korea government (MSIP).

\section{CONFLICTS OF INTEREST}

The authors have no conflicting interests.

\section{REFERENCES}

1. Abdullah LN and Chow EK (2013) Mechanisms of chemoresistance in cancer stem cells. Clin Transl Med 2, 3

2. Holen KD and Saltz LB (2001) New therapies, new directions: advances in the systemic treatment of metastatic colorectal cancer. Lancet Oncol 2, 290-297

3. O'Reilly EA, Gubbins L, Sharma S et al (2015) The fate of chemoresistance in triple negative breast cancer (TNBC). BBA Clin 3, 257-275

4. Bleau AM, Hambardzumyan D, Ozawa T et al (2009) PTEN/PI3K/Akt pathway regulates the side population phenotype and ABCG2 activity in glioma tumor stem-like cells. Cell Stem Cell 4, 226-235

5. Zhou W, Fong MY, Min Y et al (2014) Cancer-secreted miR-105 destroys vascular endothelial barriers to promote metastasis. Cancer Cell 25, 501-515

6. Hanahan D and Weinberg RA (2000) The hallmarks of cancer. Cell 100, 57-70

7. Deanfield JE, Halcox JP and Rabelink TJ (2007) Endothelial function and dysfunction: testing and clinical relevance. Circulation 115, 1285-1295

8. Lee A, Papangeli I, Park Y et al (2017) A PPARgamma- dependent miR-424/503-CD40 axis regulates inflammation mediated angiogenesis. Sci Rep 7, 2528

9. Jo HN, Kang H, Lee A et al (2017) Endothelial miR-26a regulates VEGF-Nogo-B receptor-mediated angiogenesis. BMB Rep 50, 384-389

10. Butler JM, Kobayashi $\mathrm{H}$ and Rafii S (2010) Instructive role of the vascular niche in promoting tumour growth and tissue repair by angiocrine factors. Nat Rev Cancer 10, 138-146

11. Gilbert LA and Hemann MT (2010) DNA damage-mediated induction of a chemoresistant niche. Cell 143, 355-366

12. Folberg R and Maniotis AJ (2004) Vasculogenic mimicry. APMIS 112, 508-525

13. Kirschmann DA, Seftor EA, Hardy KM, Seftor RE and Hendrix MJ (2012) Molecular pathways: vasculogenic mimicry in tumor cells: diagnostic and therapeutic implications. Clin Cancer Res 18, 2726-2732

14. Cao Z, Bao M, Miele L, Sarkar FH, Wang Z and Zhou Q (2013) Tumour vasculogenic mimicry is associated with poor prognosis of human cancer patients: a systemic review and meta-analysis. Eur J Cancer 49, 3914-3923

15. Wagenblast E, Soto M, Gutierrez-Angel S et al (2015) A model of breast cancer heterogeneity reveals vascular mimicry as a driver of metastasis. Nature 520, 358-362

16. Hendrix MJ, Seftor EA, Hess AR and Seftor RE (2003) Vasculogenic mimicry and tumour-cell plasticity: lessons from melanoma. Nat Rev Cancer 3, 411-421

17. Williamson SC, Metcalf RL, Trapani F et al (2016) Vascu logenic mimicry in small cell lung cancer. Nat Commun 7, 13322

18. Bharti R, Dey G and Mandal M (2016) Cancer development, chemoresistance, epithelial to mesenchymal transition and stem cells: A snapshot of IL-6 mediated involvement. Cancer Lett 375, 51-61

19. Dijkgraaf EM, Welters MJ, Nortier JW, van der Burg SH and Kroep JR (2012) Interleukin-6/interleukin-6 receptor pathway as a new therapy target in epithelial ovarian cancer. Curr Pharm Des 18, 3816-3827

20. Rossi JF, Lu ZY, Jourdan M and Klein B (2015) Interleukin6 as a therapeutic target. Clin Cancer Res 21, 1248-1257

21. Wang $Y$, Niu XL, Qu Y et al (2010) Autocrine production of interleukin-6 confers cisplatin and paclitaxel resistance in ovarian cancer cells. Cancer Lett 295, 110-123

22. Hong L, Sharp T, Khorsand B et al (2016) MicroRNA-200c Represses IL-6, IL-8, and CCL-5 Expression and Enhances Osteogenic Differentiation. PLoS One 11, e0160915

23. Zhang W, Shen X, Xie L, Chu M and Ma Y (2015) MicroRNA- $181 \mathrm{~b}$ regulates endotoxin tolerance by targeting IL-6 in macrophage RAW264.7 cells. J Inflamm (Lond) 12, 18

24. Hunter CA and Jones SA (2015) IL-6 as a keystone cytokine in health and disease. Nat Immunol 16, 448-457

25. Kanda T and Takahashi T (2004) Interleukin-6 and cardiovascular diseases. Jpn Heart J 45, 183-193

26. Chen SC, Chang YL, Wang DL and Cheng JJ (2006) Herbal remedy magnolol suppresses IL-6-induced STAT3 activation and gene expression in endothelial cells. $\mathrm{Br} \mathrm{J}$ Pharmacol 148, 226-232

27. Zhang GJ and Adachi I (1999) Serum interleukin-6 levels correlate to tumor progression and prognosis in metastatic breast carcinoma. Anticancer Res 19, 1427-1432 
28. Nakashima J, Tachibana M, Horiguchi $Y$ et al (2000) Serum interleukin 6 as a prognostic factor in patients with prostate cancer. Clin Cancer Res 6, 2702-2706

29. Seymour JF, Talpaz M, Cabanillas F, Wetzler $M$ and Kurzrock R (1995) Serum interleukin-6 levels correlate with prognosis in diffuse large-cell lymphoma. J Clin Oncol 13, 575-582

30. Jansson MD and Lund AH (2012) MicroRNA and cancer. Mol Oncol 6, 590-610

31. Kim J (2018) MicroRNAs as critical regulators of the endothelial to mesenchymal transition in vascular biology. BMB Rep 51, 65-72

32. Lee A, McLean D, Choi J, Kang H, Chang W and Kim J (2014) Therapeutic implications of microRNAs in pulmonary arterial hypertension. BMB Rep 47, 311-317

33. Kang $H$, Park $Y$, Lee $A$ et al (2017) Negative regulation of NOD1 mediated angiogenesis by PPARgamma-regulated miR-125a. Biochem Biophys Res Commun 482, 28-34

34. Li Z, Pan W, Shen $Y$ et al (2018) IGF1/IGF1R and microRNA let-7e down-regulate each other and modulate proliferation and migration of colorectal cancer cells. Cell Cycle 17, 1212-1219

35. Shan Y, Liu Y, Zhao L, Liu B, Li Y and Jia L (2017) MicroRNA-33a and let-7e inhibit human colorectal cancer progression by targeting ST8SIA1. Int J Biochem Cell Biol 90, 48-58

36. Xiao M, Cai J, Cai L et al (2017) Let-7e sensitizes epithelial ovarian cancer to cisplatin through repressing DNA double strand break repair. J Ovarian Res 10, 24

37. Zhu WY, Luo B, An JY et al (2014) Differential expression of miR-125a-5p and let-7e predicts the progression and prognosis of non-small cell lung cancer. Cancer Invest 32,
394-401

38. Huang P, Mao LF, Zhang ZP et al (2018) Down-Regulated miR-125a-5p Promotes the Reprogramming of Glucose Metabolism and Cell Malignancy by Increasing Levels of CD147 in Thyroid Cancer. Thyroid 28, 613-623

39. Naidu S, Shi L, Magee P et al (2017) PDGFR-modulated miR-23b cluster and miR-125a-5p suppress lung tumorigenesis by targeting multiple components of KRAS and NF-kB pathways. Sci Rep 7, 15441

40. Lu G, Ma Y, Jia C et al (2017) Reduced miR-125a levels associated with poor survival of patients with hepatocellular cancer. Oncol Lett 14, 5952-5958

41. McCall MN, Kent OA, Yu J, Fox-Talbot K, Zaiman AL and Halushka MK (2011) MicroRNA profiling of diverse endothelial cell types. BMC Med Genomics 4, 78

42. Sun M, Guo S, Yao J et al (2019) MicroRNA-125a suppresses cell migration, invasion, and regulates hyaluronic acid synthase 1 expression by targeting signal transducers and activators of transcription 3 in renal cell carcinoma cells. J Cell Biochem 120, 1894-1902

43. Zhang Y, Zhang Q, Gui L et al (2018) Let-7e inhibits TNF-alpha expression by targeting the methyl transferase EZH2 in DENV2-infected THP-1 cells. J Cell Physiol 233, 8605-8616

44. Schnegg Cl, Yang MH, Ghosh SK and Hsu MY (2015) Induction of Vasculogenic Mimicry Overrides VEGF-A Silencing and Enriches Stem-like Cancer Cells in Melanoma. Cancer Res 75, 1682-1690

45. van der Schaft DW, Seftor RE, Seftor EA et al (2004) Effects of angiogenesis inhibitors on vascular network formation by human endothelial and melanoma cells. J Natl Cancer Inst 96, 1473-1477 